\title{
Efeitos tóxicos de fármacos antidiabéticos como poluentes sob os parâmetros biológicos do peixe zebrafish (Danio rerio)
}

\author{
Toxic effects of antidiabetic drugs as pollutants on the biological parameters of the fish \\ zebrafish (Danio rerio)
}

\section{Efectos tóxicos de los fármacos antidiabéticos como contaminantes bajo los parámetros biológicos del pez cebra (Danio rerio)}

Niely Priscila Correia da Silva ${ }^{1}$, Tiago Queiroz da Mota Bittencourt ${ }^{1}$, Thamiris Pinheiro Santos ${ }^{1}$, Amanda Rodrigues dos Santos Magnabosco ${ }^{1}$, Marília Cordeiro Galvão da Silva ${ }^{1}$, Jadson Freitas da Silva ${ }^{1}$, Paulo Eduardo da Silva Bastos ${ }^{1}$, Renata Meireles Oliveira Padilha ${ }^{1}$, Marilia Ribeiro Sales Cadena ${ }^{1}$, Pabyton Gonçalves Cadena ${ }^{1 *}$.

\section{RESUMO}

Objetivo: Avaliar os efeitos tóxicos causados pela exposição a antidiabéticos em concentrações encontradas no meio ambiente sob os parâmetros biológicos de Danio rerio. Métodos: O desenvolvimento embrionário e larval foi avaliado sob exposição à metformina, glibenclamida, glimepirida e gliclazida de 1 a 144 hpf. Os possíveis efeitos tóxicos foram divididos em letais, subletais e teratogênicos. Resultados: Todos os antidiabéticos causaram uma mortalidade baixa de menos de $10 \%$ causada pela ausência de batimento cardíaco. O efeito subletal em animais expostos à metformina foi a ausência de inflação da bexiga natatória nas larvas. A glibenclamida apresentou efeitos subletais e teratogênicos em todas as concentrações, sendo considerada a droga mais tóxica. A glimepirida causou uma redução na pigmentação e a gliclazida teve a toxicidade mais baixa, mas foi a única droga que causou retardo de crescimento. Conclusão: Concluímos que os fármacos avaliados apresentam baixa toxicidade. Encontramos toxicidade diferente nas duas fases do desenvolvimento do peixe-zebra, sendo as larvas as mais afetadas. Como a metformina é o antidiabético mais comum encontrado no ambiente aquático, recomendamos o monitoramento desse composto para tentar mitigar possíveis impactos ambientais.

Palavras-chave: Ecotoxicologia, Glicemia, Peixe, Biguanidas, Sulfoniluréias.

\section{ABSTRACT}

Objective: To evaluate the toxic effects caused by exposure to antidiabetics at concentrations found in the environment under the biological parameters of Danio rerio. Methods: Embryonic and larval development was evaluated under exposure to metformin, glibenclamide, glimepiride and gliclazide from 1 to $144 \mathrm{hpf}$. Possible toxic effects were divided into lethal, sublethal and teratogenic. Results: All antidiabetics caused a low mortality of less than $10 \%$ caused by the absence of a heartbeat. The sublethal effect in metformin-exposed animals was the absence of swim bladder inflation in the larvae. Glibenclamide showed sublethal and teratogenic effects at all concentrations, being considered the most toxic drug. Glimepiride caused a reduction in pigmentation and gliclazide had the lowest toxicity, but it was the only drug that caused growth retardation. Conclusion: We conclude that the drugs evaluated have low toxicity. We found different toxicity in the two stages of zebrafish development, with larvae being the most affected. As metformin is the most common antidiabetic found in the aquatic environment, we recommend monitoring this compound to try to mitigate possible environmental impact.

Keywords: Ecotoxicology, Blood glucose, Fish, Embryos, Sulfonylureas.

1 Universidade Federal Rural de Pernambuco (UFRPE), Recife - PE. *E-mail: pabyton.cadena@ufrpe.br 


\section{RESUMEN}

Objetivo: Evaluar los efectos tóxicos provocados por la exposición a fármacos antidiabéticos en concentraciones encontradas en el ambiente bajo los parámetros biológicos de Danio rerio. Métodos: Se evaluó el desarrollo embrionario y larvario bajo exposición a metformina, glibenclamida, glimepirida y gliclazida de 1 a 144 hpf. Los posibles efectos tóxicos se dividieron en letales, subletales y teratogénicos. Resultados: Todos los antidiabéticos causaron una baja mortalidad de menos del 10\% como lo causado la ausencia de latidos cardíacos. El efecto subletal en los animales expuestos a metformina fue la ausencia de inflado de la vejiga natatoria en las larvas. La glibenclamida mostró efectos subletales y teratogénicos en todas las concentraciones, considerado él fármaco más tóxico. La glimepirida provocó una reducción de la pigmentación y la gliclazida tuvo la toxicidad más baja, pero fue el único fármaco que provocó un retraso en el crecimiento. Conclusión: Concluimos que los fármacos evaluados presentado baja toxicidad. Encontramos diferente toxicidad en las dos etapas del desarrollo del pez cebra, siendo las larvas las más afectadas. Como la metformina es el antidiabético más que se encuentra en el medio acuático, recomendamos monitorear este compuesto para tratar de mitigar los posibles daños ambientales.

Palabras clave: Ecotoxicología, Glucosa, Pescado, Embriones, Sulfonilureas.

\section{INTRODUÇÃO}

O diabetes é uma doença crônica que tem afetado milhões de pessoas no mundo e está relacionado com alterações nas taxas de glicemia no sangue (SIMPSON SH, et al., 2014). A World Health Organization (WHO) relata que a produção insuficiente ou o uso inadequado de insulina produzida pelo pâncreas caracteriza 0 diabetes como um dos mais sérios problemas de saúde. Pessoas com diabetes podem apresentar complicações como nefropatia, retinopatia e neuropatia (WHO, 2019).

Dentre todos os tipos de diabetes, o tipo 2 é responsável por $90 \%$ a $95 \%$ dos casos, e tem crescido de forma significativa devido alguns fatores como o crescimento populacional e aumento da expectativa de vida, alimentação inadequada, sedentarismo e obesidade, favorecendo o desenvolvimento da doença (CUBAS MR, et al., 2013; WHO, 2019).

O principal tratamento do diabetes é feito através de antidiabéticos orais, que tem como objetivo o controle da glicemia como a metformina (Metf), uma biguanida muito usada no tratamento do diabetes tipo 2, sendo o principal efeito o aumento à sensibilidade a insulina e redução da taxa de gliconeogênese hepática (SALES WB, et al., 2015; MARTíNEZ-VIVEROS EMG, et al., 2018). Ainda, o mecanismo de ação da metformina mantém-se indefinido, visto que os efeitos deste antidiabético certamente são pleiotrópicos (FLOREZ JC, 2017; FLORY J e LIPSKA K, 2019).

As sulfoniluréias como a glibenclamida (Glib), glimepirida (Glim) e gliclazida (Glic) também são muito recomendadas a pacientes diabéticos por reduzirem efetivamente a glicemia plasmática e o risco de complicações microvasculares como a nefropatia e retinopatia (SIMPSON SH, et al., 2014).

O mecanismo de ação das sulfoniluréias é independente da concentração de glicose no sangue, e agem inibindo a abertura dos canais de potássio que são sensíveis às moléculas de ATP, permitindo uma despolarização das células $\beta$-pancreáticas o que induz o influxo de íons cálcio aumentando a liberação de insulina pelos grânulos de secreção (KALRA S e GUPTA Y, 2015; WEI LV, et al., 2020). Elas também se aderem a proteína Epac 2 (proteína de troca diretamente ativada por $\mathrm{AMP}_{\mathrm{C}} 2$ ), e interagem com outras proteínas para ampliar o número de grânulos de insulina (KALRA S e GUPTA Y, 2015).

A International Diabetes Federation (IDF) em 2019 registrou no Brasil 16,8 milhões de casos de diabetes em adultos, colocando o país na quinta posição em número de portadores da doença (IDF, 2019). Este grande número de casos aumenta a ocorrência dos fármacos residuais comumente usados no tratamento do diabetes em efluentes. Estes podem ser provenientes de indústrias farmacêuticas, hospitais, liberação através das fezes e urina associado ao tratamento inadequado de esgotos domésticos que permitem a chegada destes antidiabéticos ao meio ambiente (MARKIEWICZ M, et al., 2017). Isto leva a preocupação quanto aos possíveis efeitos tóxicos que podem apresentar aos seres humanos e animais incluindo os peixes, como a desregulação endócrina (NIEMUTH NJ e KLAPER RD, 2018). 
Devido à presença desses antidiabéticos no ambiente aquático (TERNES T, et al., 2001; ALAHMAD WR e ALAWI MA, 2010; AL-QAIM FF, 2016; MARKIEWICZ M, et al., 2017; NIEMUTH NJ e KLAPER RD, 2018), é de importância a realização de testes toxicológicos utilizando peixes como modelo animal para avaliar os efeitos da exposição destes fármacos antidiabéticos que podem ser encontrados no meio ambiente (SOUZA $E Q$, et al., 2019).

Danio rerio (zebrafish), é uma das espécies mais utilizadas em pesquisas laboratoriais, por apresentar facilidade em seu manejo, uma alta fecundidade, embriões transparentes e relativamente grandes com um rápido desenvolvimento, o que possibilita que analises de doenças possam ser feitas durante diferentes fases de seu desenvolvimento nas condições laboratoriais. Também possui $70 \%$ de similaridade genética com humanos, o que favorece estudos relacionados a doenças (HOWE K, et al., 2013; SPIRITA SV e AHILA AJ, 2015; TABASSUM N, et al., 2015).

Essas características tornam o zebrafish um modelo animal viável, além de apresentar aspectos considerados para atender os critérios do três R's (substituição, refinamento e redução) estabelecidos pelo Conselho Nacional de Controle de Experimentação Animal (CONCEA, 2014), que tem por finalidade o reconhecimento de métodos que visem a substituição, refinamento ou redução do uso de animais em atividades de pesquisas (BRASIL, 2014; GHENO EM, et al., 2016).

Anteriormente, nosso grupo avaliou os efeitos tóxicos de metformina e glibenclamida em relação ao ganho de peso, comportamento animal, à glicemia e à mortalidade de adultos de $D$. rerio e Hemigrammus caudovittatus (SOUZA EQ, et al., 2019). Agora, o objetivo do trabalho foi avaliar os efeitos tóxicos causados pela exposição a fármacos antidiabéticos comumente usados no Brasil em concentrações encontradas no ambiente sob os parâmetros biológicos do Danio rerio.

\section{MÉTODOS}

Os experimentos foram realizados no Laboratório de Ecofisiologia e Comportamento Animal (LECA) da Universidade Federal Rural de Pernambuco (UFRPE), com métodos previamente aprovados pela Comissão de Ética no Uso de Animais (CEUA) licenças 40/2017 (Metformina), 101/2018 (Glibenclamida) e 026/2019 (Gliclazida e Glimepirida).

Animais adultos da espécie Danio rerio foram adquiridos em criadouros comerciais e mantidos no laboratório em quarentena para detectar e confirmar a ausência de patógenos ou doenças, sendo acondicionados em aquários de 80 litros, aerados com biofiltro JEBO 503, com fotoperíodo 14/10 h (claro/escuro) e temperatura controlada $\left(26 \pm 1^{\circ} \mathrm{C}\right)$. A água foi tratada com adição de tiossulfato de sódio para redução do cloro e hidróxido de sódio para a manutenção do pH em 7,0 \pm 0,5.

Para a obtenção dos ovos, foi utilizado o protocolo da Organização para a Cooperação e Desenvolvimento Econômico (OCDE) os animais adultos foram separados em aquários de reprodução (patente BR 202016 017042 2), utilizando machos e fêmeas na proporção de 2:1. Após desova, foram recolhidos os ovos e observados por meio de microscópio óptico de luz (com lâmpada LED) a 1 hpf (Hora pós-fertilização) (BITTENCOURT TQM, et al., 2018; CADENA PG, et al., 2020). Foram utilizados ovos cuja desova apresentava mais de $90 \%$ de viabilidade (OCDE, 2013).

Para formação dos grupos experimentais foram utilizados ovos fertilizados (desenvolvimento normal da blástula), enquanto os não fertilizados (com injurias, clivagens irregulares, ou malformações) foram descartados. Os ovos foram aleatoriamente acondicionados em potes estéreis de poliestireno com capacidade de $80 \mathrm{~mL}$, mantidos em incubadora (patente BR 1020180150 4) com temperatura controlada de $26 \pm 1^{\circ} \mathrm{C}, \mathrm{pH} 7,5 \pm 0,5$ e fotoperíodo $14 / 10 \mathrm{~h}$ (claro/escuro).

As concentrações dos antidiabéticos utilizados nos experimentos foram baseadas nas concentrações encontradas no ambiente aquático sendo $40 \mu \mathrm{g} / \mathrm{L}$ de metformina (Metf), 0,013 $\mu \mathrm{g} / \mathrm{L}$ de glibenclamida (Glib), $103 \mu \mathrm{g} / \mathrm{L}$ de glimepirida (Glim) e 0,015 $\mu \mathrm{g} / \mathrm{L}$ de gliclazida (Glic) (TERNES T, et al., 2001; ALAHMAD WR e ALAWI MA, 2010; AL-QAIM FF, 2016; NIEMUTH NJ e KLAPER RD, 2018). 
Para o preparo das soluções de trabalho de Metf, Glib e Glim, amostras comerciais foram maceradas e dissolvidas em água destilada. A Glic foi primeiramente dissolvida em acetona a 1\% (v/v), em seguida a solução foi diluída com água destilada para evaporação da acetona. Com isso, foi possível obter as seguintes

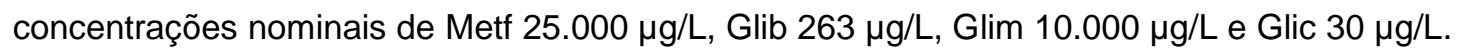

A partir destas soluções de trabalho foi feita a diluição com água declorada para a obtenção das soluções teste usada nos experimentos com as seguintes concentrações para cada composto: 20, 40 (ambiental), 100, 600 e $1000 \mu \mathrm{g} / \mathrm{L}$ para Metf; 0,007, 0,013 (ambiental), ,320, 3,200 e 6,400 $\mu \mathrm{g} / \mathrm{L}$ para Glib; 52, 103 (ambiental), 254, 2535 e $5071 \mu \mathrm{g} / \mathrm{L}$ para a Glim; e 0,008, 0,015 (ambiental), 0,037, 0,185 e 0,369 $\mu \mathrm{g} / \mathrm{L}$ para Glic.

As concentrações usadas nas soluções teste foram aumentadas de forma arbitrária em relação à ambiental, levando em consideração um possível cenário de aumento do descarte destes antidiabéticos no ambiente ou acidente com liberação de grande quantidade. Os testes de toxicidade foram realizados de acordo com a OCDE (2013) com planejamento estatístico realizado por parcela subdividida (parcela $=$ concentração do antidiabético; subparcela $=$ tempo), tendo os dados expressos de acordo com à dispersão das frequências (Absoluta e Relativa).

Os animais foram expostos as diferentes soluções teste sendo 5 concentrações por antidiabético com 30 animais por grupo experimental ( $3 \times 10$ animais por pote de poliestireno) e grupo controle, durante 1 até 144 hpf (horas pós-fertilização). As soluções teste foram renovadas diariamente com controle das condições abióticas conforme descrito acima (OCDE, 2013). Os resíduos gerados durante o experimento passaram por tratamento em processo oxidativo avançado em reator utilizando foto-oxidação $\mathrm{UV} \mathrm{e} \mathrm{H}_{2} \mathrm{O}_{2}$ antes do descarte (SILVA MCG, et al., 2019).

O desenvolvimento embrionário (24 - 48 hpf) e larval (72 -144 hpf) de $D$. rerio foi usado como variável resposta e avaliado sob exposição a diferentes concentrações de Metf, Glib, Glim, Glic e grupo controle. Com o auxílio de um microscópio óptico (400x, 1000x), os embriões e larvas de $D$. rerio foram fotografados para identificação de possíveis efeitos tóxicos. A avaliação da toxicidade foi dividida em três categorias de efeitos, de acordo com Lammer E, et al. (2009), OCDE (2013) e Silva MCG, et al. (2019) modificado sendo efeitos de letalidade, efeitos subletais (não permanentes) e efeitos de teratogenicidade (malformações).

Nos efeitos de letalidade, a mortalidade foi observada diariamente, sendo apresentada em porcentagem (\%). A determinação da letalidade foi realizada pela coagulação, falta de eclosão, ausência de batimentos cardíacos, sem formação de somitos e cauda não destacada. Nos efeitos subletais, a frequência cardíaca ( $\mathrm{FrC})$ foi mensurada através da contagem manual dos batimentos cardíacos (SILVA MCG, et al., 2019). Também foram avaliados a área de coagulação $(A c)$, redução na pigmentação $(R p)$, edema de pericárdio (EP) e edema do saco vitelínico (ES). Os efeitos de teratogenicidade analisados foram malformação dos olhos (Mf), retardo do crescimento (Rt), ausência de inflação da bexiga natatória (AB), deformação da coluna (DL), deformação da cauda (DC) e deformação do saco vitelínico (DS).

Os dados dos efeitos de letalidade, subletais e de teratogenicidade foram agrupados por resposta dicotômica (presente ou ausente). Em seguida, analisados pela frequência relativa cujos dados foram apresentados em escala de cores que avaliou os animais afetados e permitiu a categorização da população em estratos onde: "Verde" - Ausência de alterações; (Amarelo) até $25 \%$ dos animais apresentaram o efeito "leve"; (Laranja) > 25 até $50 \%$ dos animais apresentaram o efeito "moderado"; (Vermelho) $>51 \%$ até $75 \%$ dos animais apresentaram o efeito "severo"; (Preto) $>76 \%$ dos animais apresentaram o efeito "muito severo". Como critérios de validação, os animais do grupo controle não apresentaram qualquer dos efeitos subletais e teratogênicos analisados e em cada teste a mortalidade do grupo controle não excedeu $10 \%$, o que poderia invalidar todo o ensaio (OCDE, 2013).

Para a frequência cardíaca, foi determinada a média e desvio padrão de cada grupo. Os resultados foram analisados por one-Way ANOVA. Quando a diferença foi significativa, as médias foram comparadas através do teste de Tukey sendo $p<0,05$. Para as análises estatísticas, foi utilizado o software Origin Pro Academic 2015 (Origem Lab. Northampton, MA, EUA). 


\section{RESULTADOS}

A exposição dos animais aos antidiabéticos revelou baixa mortalidade, sempre inferior a 10\%, constatada pela ausência de batimentos cardíacos. Portanto, os efeitos tóxicos observados foram mais relacionados aos efeitos subletais e de teratogenicidade. Esses efeitos podem ser visualizados na (Figura 1).

Na Figura 1 são observados exemplos de efeitos subletais e teratogênicos em embriões e larvas de $D$. rerio após exposição a diferentes concentrações de Metf, Glib, Glim e Glic. Na figura A e B são exemplos de animais saudáveis do grupo controle. $\mathrm{Em} \mathrm{C}$ e $\mathrm{D}$ exemplos de efeitos subletais que foram visualizados durante a coleta dos dados, sendo eles redução na pigmentação, edema de pericárdio e edema do saco vitelínico em embriões e larvas com 96 hpf. Já as figuras $E$ e $F$ exemplificam efeitos teratogênicos como malformação dos olhos, ausência de bexiga natatória, deformação de coluna, deformação de cauda, deformação do saco vitelínico e retardo no crescimento em larvas com 96 e 144 hpf, respectivamente.

Figura 1 - Efeitos subletais e de teratogenicidade tipicamente observados em embriões e larvas de Danio rerio após exposição aos antidiabéticos metformina, glibenclamida, glimepirida e gliclazida.
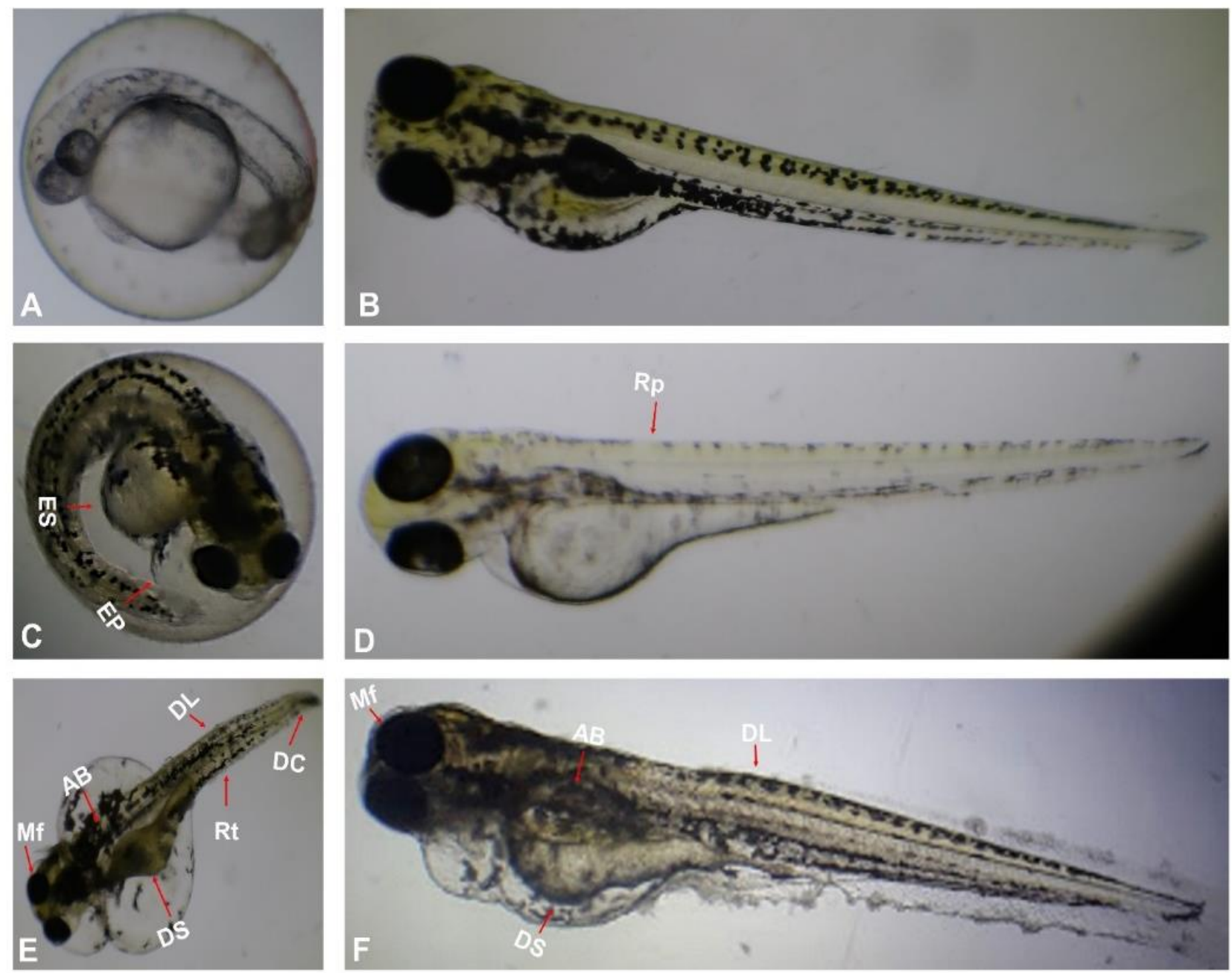

Notas: A e B representam exemplos de animais saudáveis do grupo controle. C e D representam efeitos subletais em embriões e larvas com $72 \mathrm{hpf}$ e $96 \mathrm{hpf}$. E e $\mathrm{F}$ representam efeitos de teratogenicidade em larvas com 96 e 144 hpf, respectivamente. Legenda: Rp - redução na pigmentação; EP - edema de pericárdio; ES - edema do saco vitelínico; Mf - malformação dos olhos; AB - ausência de inflação da bexiga natatória; DL deformação na coluna; DC - deformação na cauda; DS- deformação do saco vitelínico; Rt - retardo no crescimento.

Fonte: Silva NPC, et al., 2021. 
A Metf no efeito de desenvolvimento subletal apresentou apenas edema de pericárdio (até $25 \%$ dos animais) nos embriões em 24 hpf e redução na pigmentação (até 50\% dos animais) nas larvas entre 72 e 96 hpf (Figura 2). O efeito de teratogenicidade revelou toxicidade nas larvas entre 96 e 120 hpf como ausência de inflação da bexiga natatória (de $25 \%$ até $100 \%$ dos animais) observado em todas as concentrações e deformação na cauda (até 25\% dos animais) apenas em 144 hpf (Figura 2).

$\mathrm{Na}$ frequência cardíaca foi observado aumento e redução significativa $(p<0,05)$, mas sem relação com a dose e com o tempo (Tabela 1). Como observamos baixa mortalidade neste grupo, provavelmente esta variação pode ser considerada transitória e de baixa toxicidade.

Figura 2 - Efeitos de desenvolvimentos subletais e de teratogenicidade em embriões (24 - 48 hpf) e larvas (72 - 144 hpf) de zebrafish (Danio rerio) expostos a diferentes concentrações de metformina, com avaliações entre 24 - 144 horas pós-fertilização (hpf).

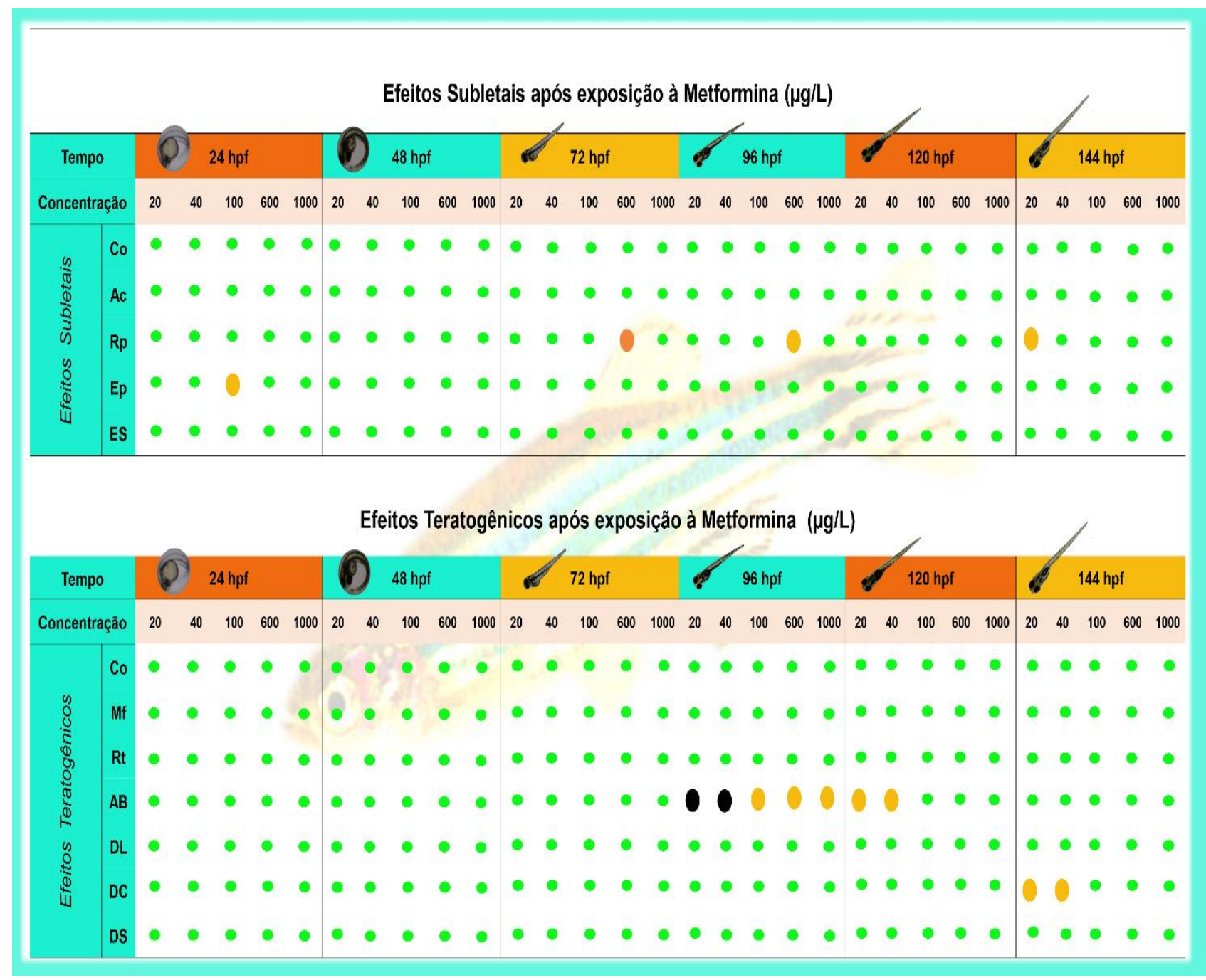

Legenda: Co - Grupo controle sem exposição aos antidiabéticos; (Verde) não foi observado o efeito nos animais; (Amarelo) até $25 \%$ dos animais apresentaram o efeito (leve); (Laranja) $>25$ até $50 \%$ dos animais apresentaram o efeito (moderado); (Vermelho) $>50 \%$ até $75 \%$ dos animais apresentaram o efeito (severo); (Preto) $>75 \%$ dos animais apresentaram o efeito (muito severo); Ac - área de coagulação, Rp - redução na pigmentação, EP - edema de pericárdio, ES- edema do saco vitelínico, Mf - malformação dos olhos; Rt retardo do crescimento; $A B$ - ausência de inflação da bexiga natatória; DL - deformação da coluna; DC deformação da cauda; DS - deformação do saco vitelínico.

Fonte: Silva NPC, et al., 2021. 
Após exposição a Glib no efeito de desenvolvimento subletal foi observado nas larvas redução na pigmentação (até 50\% dos animais) a partir das $72 \mathrm{hpf}$, edema de pericárdio e edema de saco vitelínico em até $25 \%$ dos animais em $96 \mathrm{hpf}$ (Figura 3). O efeito de teratogenicidade revelou toxicidade nas larvas a partir de 96 hpf como malformação nos olhos, deformação da cauda, deformação da coluna e deformação do saco vitelínico em até $25 \%$ dos animais (Figura 3). A ausência de inflação da bexiga natatória foi o efeito mais observado em todas as concentrações com mais de $25 \%$ da população afetada.

Na frequência cardíaca foi observado pequeno aumento e redução $(p<0,05)$ mas sem relação com a dose e com o tempo (Tabela 1). Entretanto este efeito foi menos observado em comparação com Metf indicando que Glib apresenta menos efeito tóxico sobre a frequência cardíaca.

Figura 3 - Efeitos de desenvolvimentos subletais e de teratogenicidade em embriões (24 - 48 hpf) e larvas (72 - 144 hpf) de zebrafish (Danio rerio) expostos a diferentes concentrações de glibenclamida, com avaliações entre 24 - 144 horas pós-fertilização (hpf).
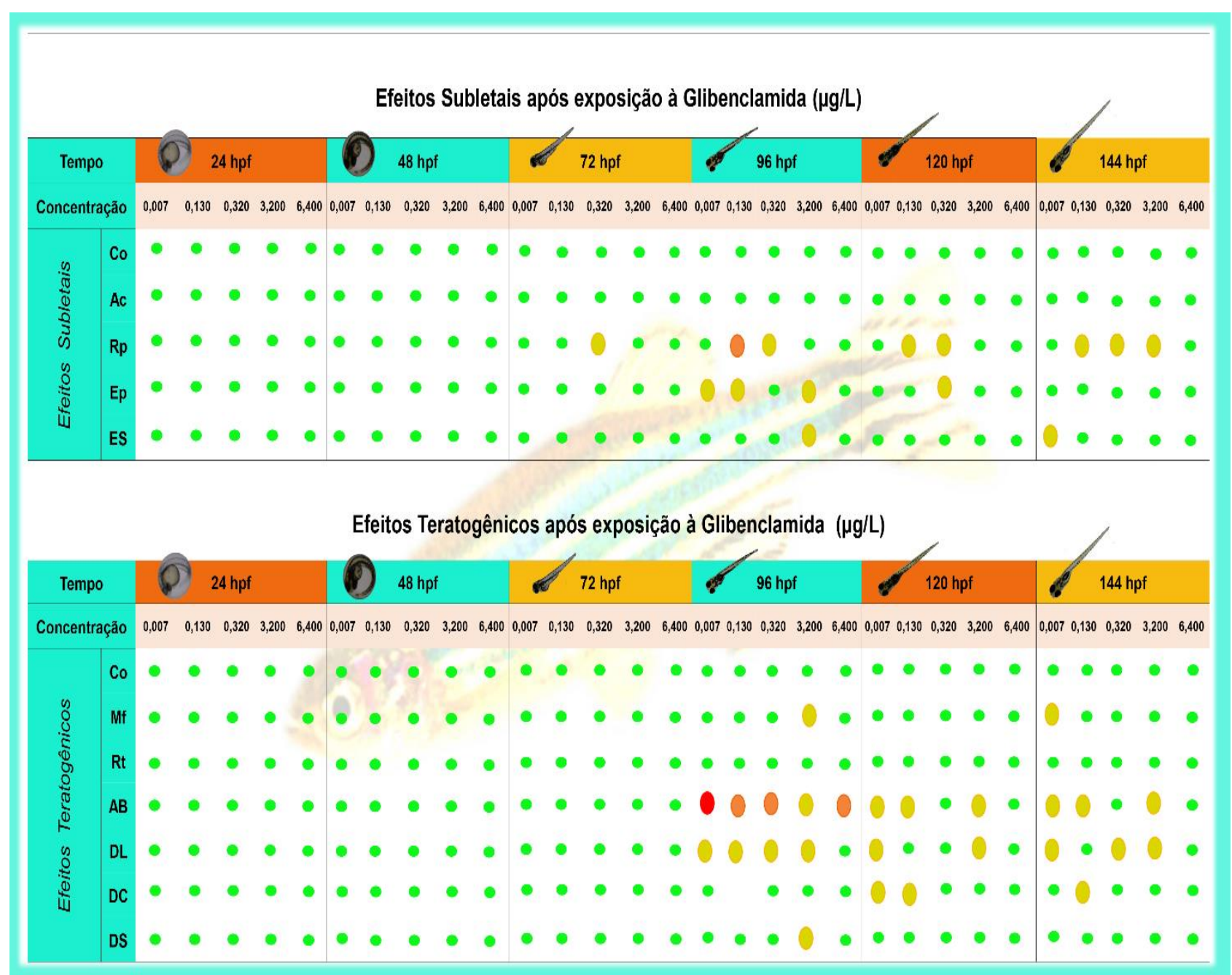

Legenda: Co - Grupo controle sem exposição aos antidiabéticos; (Verde) não foi observado o efeito nos animais; (Amarelo) até 25\% dos animais apresentaram o efeito (leve); (Laranja) $>25$ até $50 \%$ dos animais apresentaram o efeito (moderado); (Vermelho) > 50\% até $75 \%$ dos animais apresentaram o efeito (severo); (Preto) $>75 \%$ dos animais apresentaram o efeito (muito severo); Ac - área de coagulação, Rp - redução na pigmentação, EP - edema de pericárdio, ES- edema do saco vitelínico, Mf - malformação dos olhos; Rt retardo do crescimento; $A B$ - ausência de inflação da bexiga natatória; DL - deformação da coluna; DC deformação da cauda; DS - deformação do saco vitelínico.

Fonte: Silva NPC, et al., 2021. 
$\mathrm{Na}$ Glim no efeito de desenvolvimento subletal foi observado redução na pigmentação com mais de $25 \%$ dos animais afetados, edema de pericárdio entre 24 e 72 hpf e edema de saco vitelínico nas 48 hpf (até 25\% dos animais) (Figura 4). $O$ efeito de teratogenicidade revelou toxicidade a partir de $48 \mathrm{hpf}$ como deformação da cauda, deformação da coluna e deformação do saco vitelínico (até $50 \%$ dos animais) (Figura 4).

A frequência cardíaca dos animais expostos Glim apresentou aumento $(p<0,05)$ no embrião as 24 hpf e da larva a $120(p<0,05)$ (Tabela 1). Isto pode indicar toxicidade diferente em dois períodos do desenvolvimento do animal.

Figura 4 - Efeitos de desenvolvimentos subletais e de teratogenicidade em embriões (24 - 48 hpf) e larvas (72 - 144 hpf) de zebrafish (Danio rerio) expostos a diferentes concentrações de glimepirida, com avaliações entre 24 - 144 horas pós-fertilização (hpf).

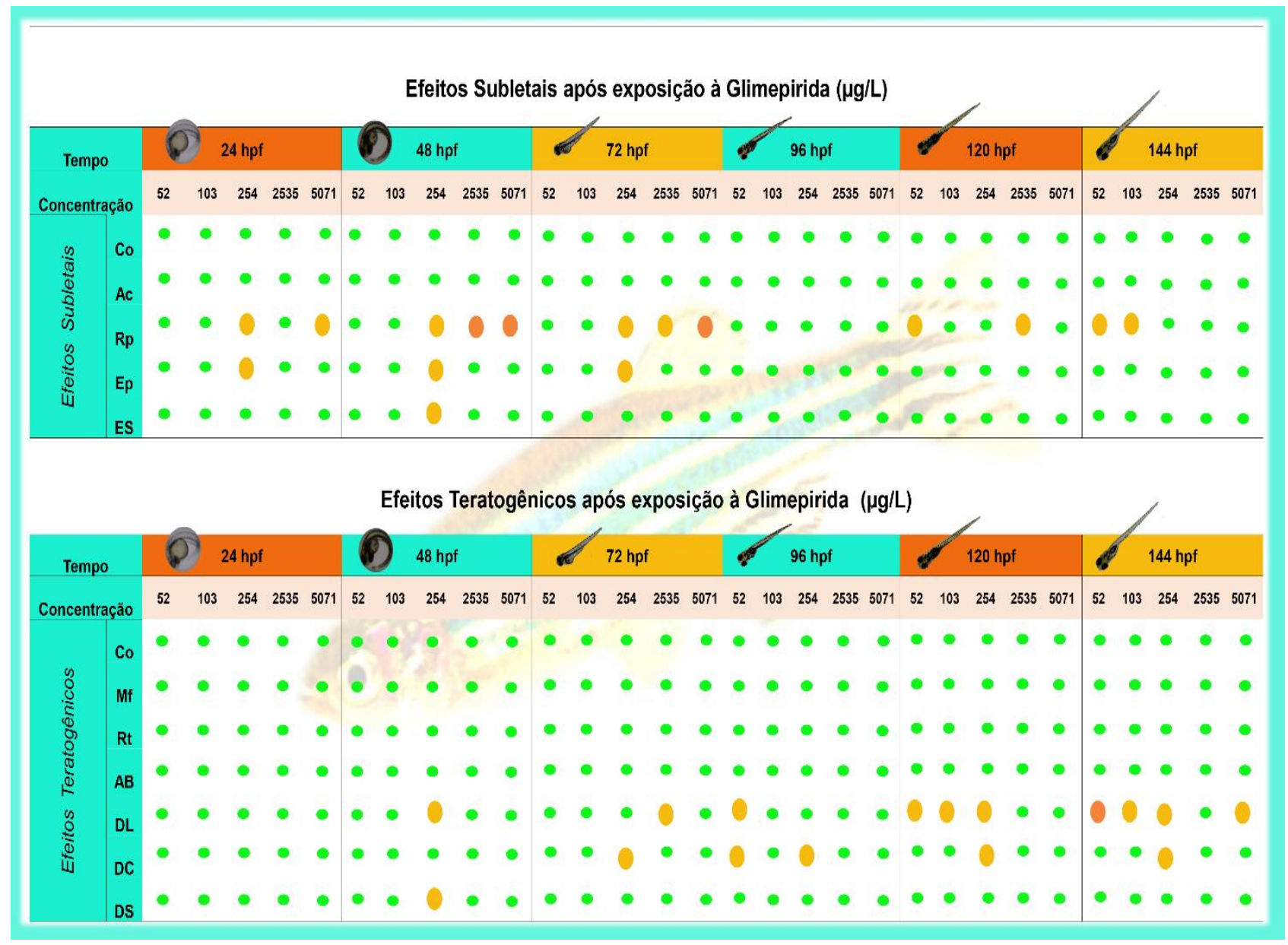

Legenda: Co - Grupo controle sem exposição aos antidiabéticos; (Verde) não foi observado o efeito nos animais; (Amarelo) até $25 \%$ dos animais apresentaram o efeito (leve); (Laranja) $>25$ até $50 \%$ dos animais apresentaram o efeito (moderado); (Vermelho) $>50 \%$ até $75 \%$ dos animais apresentaram o efeito (severo); (Preto) $>75 \%$ dos animais apresentaram o efeito (muito severo); Ac - área de coagulação, Rp - redução na pigmentação, EP - edema de pericárdio, ES- edema do saco vitelínico, Mf - malformação dos olhos; Rt retardo do crescimento; $A B$ - ausência de inflação da bexiga natatória; DL - deformação da coluna; DC deformação da cauda; DS - deformação do saco vitelínico.

Fonte: Silva NPC, et al., 2021.

A Glic apresentou, no efeito de desenvolvimento subletal, edema de pericárdio em 24 hpf, edema de saco vitelínico a partir de $48 \mathrm{hpf}$ e redução na pigmentação em $144 \mathrm{hpf}$ com até $25 \%$ dos animais afetados (Figura 5). O efeito de teratogenicidade revelou toxicidade como malformação dos olhos, retardo do crescimento, 
deformação da coluna, deformação da cauda, deformação do saco vitelínico e ausência de inflação da bexiga natatória com até $25 \%$ dos animais afetados (Figura 5). A frequência cardíaca no período das $24 \mathrm{hpf}$ apresentou um aumento $(p<0,05)$ e uma redução $(p<0,05)$ nos estágios mais avançados de desenvolvimento (Tabela 1). Isso demonstra que cada antidiabético apresentou toxicidade diferente em relação a frequência cardíaca, sendo Glib o menos tóxico. Entretanto, em todos os casos a variação foi pequena.

Figura 5 - Efeitos de desenvolvimentos subletais e de teratogenicidade em embriões (24 - 48 hpf) e larvas (72 - 144 hpf) de zebrafish (Danio rerio) expostos a diferentes concentrações de gliclazida, com avaliações entre 24 - 144 horas pós-fertilização (hpf).

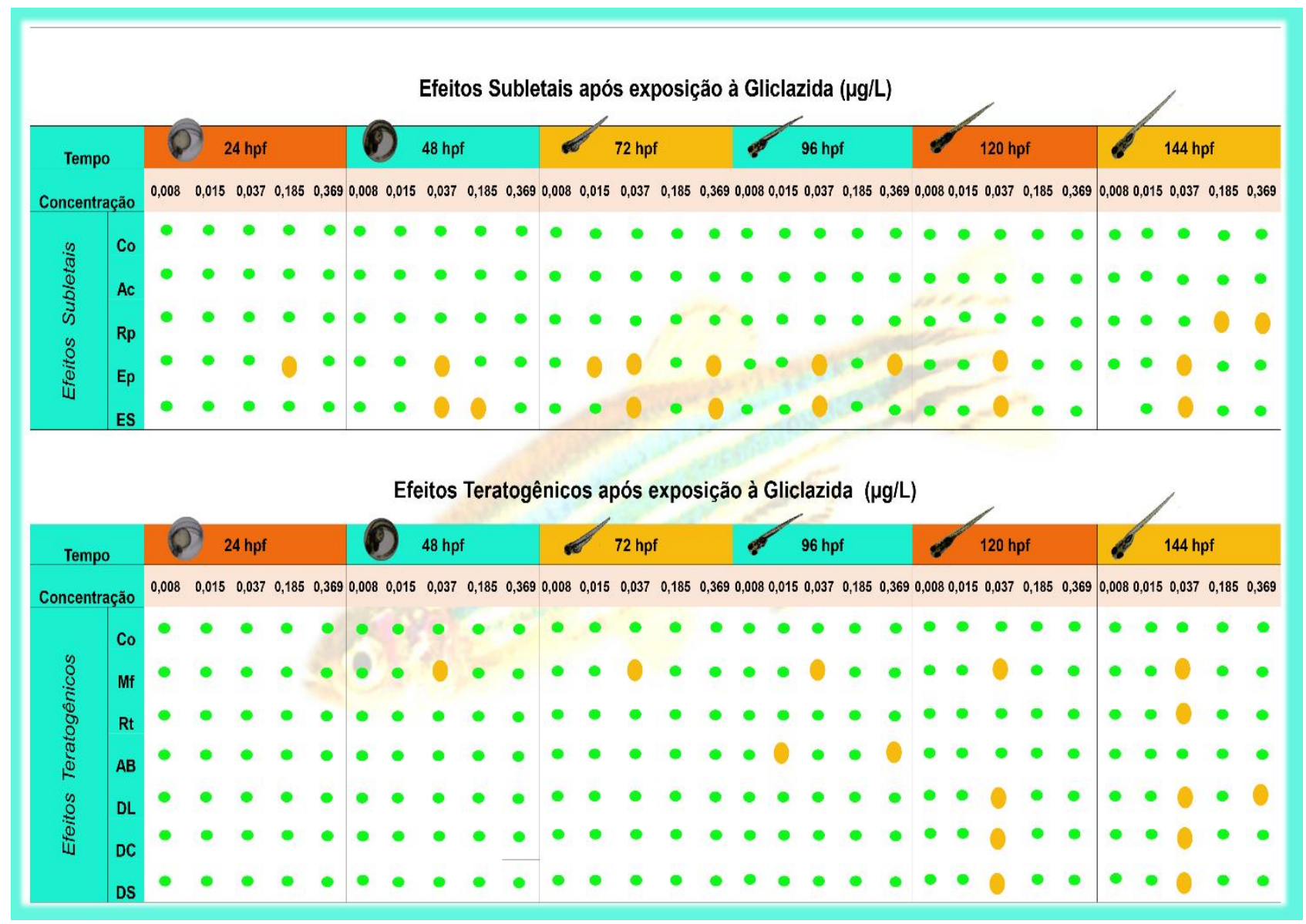

Legenda: Co - Grupo controle sem exposição aos antidiabéticos; (Verde) não foi observado o efeito nos animais; (Amarelo) até 25\% dos animais apresentaram o efeito (leve); (Laranja) > 25 até $50 \%$ dos animais apresentaram o efeito (moderado); (Vermelho) > 50\% até 75\% dos animais apresentaram o efeito (severo); (Preto) $>75 \%$ dos animais apresentaram o efeito (muito severo); Ac - área de coagulação, Rp - redução na pigmentação, EP - edema de pericárdio, ES- edema do saco vitelínico, Mf - malformação dos olhos; Rt retardo do crescimento; $A B$ - ausência de inflação da bexiga natatória; DL - deformação da coluna; DC deformação da cauda; DS - deformação do saco vitelínico.

Fonte: Silva NPC, et al., 2021.

Observou-se em todos os antidiabéticos baixa toxicidade constatada pela baixa mortalidade. Já os efeitos de desenvolvimento subletal e de teratogenicidade não tiveram relação com a concentração e sim com o período de desenvolvimento do animal sendo o período larval o mais afetado (Figura 2, 3, 4 e 5). Embora alterações significativas na frequência cardíaca tenham sido observadas em distintos períodos, eatas não estão relacionadas com as concentrações utilizadas (Tabela 1). 
Tabela 1 - Frequência cardíaca observada durante os experimentos em embriões e larvas de zebrafish após exposição aos antidiabéticos metformina, glibenclamida, glimepirida e gliclazida.

\begin{tabular}{|c|c|c|c|c|c|c|}
\hline \multirow{2}{*}{ Grupo } & \multicolumn{5}{|c|}{ Tempo } & \multirow[b]{2}{*}{144 hpf } \\
\hline & $24 \mathrm{hpf}$ & $48 \mathrm{hpf}$ & $72 \mathrm{hpf}$ & 96 hpf & $120 \mathrm{hpf}$ & \\
\hline Controle & $81 \pm 22$ & $156 \pm 21$ & $182 \pm 24$ & $204 \pm 20$ & $206 \pm 21$ & $216 \pm 18$ \\
\hline Metf $20 \mu \mathrm{g} / \mathrm{L}$ & $70 \pm 11^{*}$ & $138 \pm 7^{*}$ & $163 \pm 7^{*}$ & $165 \pm 6^{*}$ & $192 \pm 12^{\star}$ & $207 \pm 8$ \\
\hline Metf $40 \mu \mathrm{g} / \mathrm{L}$ & $97 \pm 9^{*}$ & $156 \pm 5$ & $192 \pm 14$ & $176 \pm 6^{*}$ & $183 \pm 11^{*}$ & $212 \pm 10$ \\
\hline Metf $100 \mu \mathrm{g} / \mathrm{L}$ & $112 \pm 14^{\star}$ & $143 \pm 16^{*}$ & $180 \pm 8$ & $202 \pm 12$ & $201 \pm 30$ & $191 \pm 13^{*}$ \\
\hline Metf $600 \mu \mathrm{g} / \mathrm{L}$ & $85 \pm 7$ & $193 \pm 11^{*}$ & $204 \pm 13^{\star}$ & $207 \pm 20$ & $212 \pm 9$ & $193 \pm 11^{*}$ \\
\hline Metf $1000 \mu \mathrm{g} / \mathrm{L}$ & $101 \pm 10^{*}$ & $207 \pm 7^{\star}$ & $220 \pm 8^{\star}$ & $223 \pm 7^{\star}$ & $236 \pm 8^{*}$ & $213 \pm 5$ \\
\hline Glib 0,007 $\mu \mathrm{g} / \mathrm{L}$ & $81 \pm 6$ & $159 \pm 7$ & $183 \pm 18$ & $200 \pm 9$ & $213 \pm 8$ & $216 \pm 13$ \\
\hline Glib $0,130 \mu \mathrm{g} / \mathrm{L}$ & $72 \pm 8$ & $158 \pm 7$ & $173 \pm 9$ & $165 \pm 10^{*}$ & $208 \pm 8$ & $213 \pm 7$ \\
\hline Glib 0,320 $\mu \mathrm{g} / \mathrm{L}$ & $85 \pm 6$ & $140 \pm 16^{*}$ & $175 \pm 8$ & $198 \pm 12$ & $197 \pm 6$ & $218 \pm 8$ \\
\hline Glib 3,200 $\mu \mathrm{g} / \mathrm{L}$ & $78 \pm 7$ & $142 \pm 7^{*}$ & $200 \pm 12^{*}$ & $203 \pm 16$ & $220 \pm 18^{*}$ & $228 \pm 9^{*}$ \\
\hline Glib 6,400 $\mu \mathrm{g} / \mathrm{L}$ & $70 \pm 8^{*}$ & $159 \pm 5$ & $191 \pm 14$ & $213 \pm 17$ & $231 \pm 13^{*}$ & $231 \pm 6^{*}$ \\
\hline Glim 52 g/L & $99 \pm 8^{*}$ & $164 \pm 9$ & $190 \pm 11$ & $233 \pm 18$ & $217 \pm 11^{*}$ & $222 \pm 7$ \\
\hline Glim $103 \mu \mathrm{g} / \mathrm{L}$ & $103 \pm 11^{*}$ & $158 \pm 7$ & $190 \pm 7$ & $219 \pm 10$ & $240 \pm 8^{\star}$ & $206 \pm 6^{*}$ \\
\hline Glim $254 \mu \mathrm{g} / \mathrm{L}$ & $113 \pm 9^{\star}$ & $156 \pm 8$ & $188 \pm 7$ & $244 \pm 11$ & $229 \pm 10^{*}$ & $220 \pm 5$ \\
\hline Glim $2535 \mu \mathrm{g} / \mathrm{L}$ & $116 \pm 6^{*}$ & $168 \pm 8^{*}$ & $177 \pm 7$ & $227 \pm 11$ & $230 \pm 8^{*}$ & $225 \pm 7^{*}$ \\
\hline Glim $5071 \mu \mathrm{g} / \mathrm{L}$ & $123 \pm 4^{\star}$ & $174 \pm 8^{*}$ & $186 \pm 7$ & $234 \pm 13$ & $226 \pm 9^{*}$ & $222 \pm 7$ \\
\hline Glic $0,008 \mu \mathrm{g} / \mathrm{L}$ & $120 \pm 12^{*}$ & $169 \pm 8^{*}$ & $177 \pm 10$ & $185 \pm 4^{*}$ & $190 \pm 10$ & $195 \pm 8^{*}$ \\
\hline Glic $0,015 \mu \mathrm{g} / \mathrm{L}$ & $119 \pm 12^{*}$ & $147 \pm 5$ & $175 \pm 9$ & $200 \pm 6$ & $192 \pm 9$ & $196 \pm 8^{*}$ \\
\hline Glic $0,037 \mu \mathrm{g} / \mathrm{L}$ & $129 \pm 17^{\star}$ & $149 \pm 12$ & $173 \pm 18$ & $202 \pm 23$ & $188 \pm 18$ & $189 \pm 16^{*}$ \\
\hline Glic $0,185 \mu \mathrm{g} / \mathrm{L}$ & $118 \pm 9^{\star}$ & $148 \pm 9$ & $164 \pm 6^{*}$ & $204 \pm 7$ & $190 \pm 15$ & $198 \pm 7^{*}$ \\
\hline
\end{tabular}

Legenda: A diferença foi considerada significativa do controle quando ${ }^{*}=p<0,05$ pelo teste de Tukey. Siglas $\mathrm{Hpf}$ - horas pós fertilização; Metf - metformina; Glib - glibenclamida; Glim - glimepirida; Glic - gliclazida. Fonte: Silva NPC, et al., 2021.

\section{DISCUSSÃO}

Os resultados obtidos indicaram que a exposição à Metf, Glib, Glim e Glic durante 144 hpf provocaram efeitos tóxicos em $D$. rerio nos efeitos de letalidade, subletais e de teratogenicidade principalmente no período larval. Uma vez que os embriões e larvas de $D$. rerio possuem crescimento e metabolismo basal sensíveis a compostos químicos o que pode levar a alterações fisiológicas e anatômicas (LAMMER E, et al., 2009).

Os animais expostos à Metf apresentaram baixa taxa de mortalidade corroborando com Godoy $A A$, et al. (2018) que observaram este efeito em larvas de $D$. rerio em concentrações de até $600 \mathrm{mg} / \mathrm{L}$ durante $96 \mathrm{hpf}$. Entretanto, Souza EQ, et al. (2019) relataram alta mortalidade em adultos de $D$. rerio quando expostos a Metf. Esta pode alterar o metabolismo mitocondrial aumentando a mortalidade sendo, provavelmente, os adultos mais sensíveis a este efeito (NIEMUTH NJ e KLAPER RD, 2018).

A Metf é um dos antidiabéticos mais estudados durante a gestação humana, não apresentando evidência de teratogenicidade (SALES WB, et al., 2015). Isto também foi observado em nosso estudo na fase embrionária (até $48 \mathrm{hpf}$ ) de $D$. rerio. A presença do córion possui a função de proteger o embrião contra influências externas, incluindo a exposição química o que poderia justificar os resultados (LAMMER E, et al., 2009). Entretanto, após a ruptura do córion (eclosão), os peixes dão início a fase larval, o que expõe mais facilmente os animais à presença de poluentes na água sendo observado mais efeitos tóxicos no nosso estudo (OCDE, 2013).

O efeito subletal mais observado foi ausência de inflação da bexiga natatória em 96 hpf. Entretanto, este efeito tóxico reduziu à medida que os animais cresceram, não sendo mais observado em $144 \mathrm{hpf}$. Stinckens E, et al. (2018) relataram que este efeito pode estar relacionado com a redução na secreção dos hormônios da tireoide. Metf é conhecido como um disruptor endócrino para peixes e apresenta efeito redutor na secreção 
de hormônios da tireoide (NIEMUTH NJ e KLAPER RD, 2018). Em estudo realizado por Alla LNR, et al. (2021), a exposição química à Metf alterou o comportamento natatório de $D$. rerio em uma concentração de $10 \mathrm{nM}$ mostrando toxicidade do antidiabético em pequenas concentrações como observado em nosso estudo.

A exposição à Glib revelou baixa taxa de mortalidade nos animais. Entretanto, Souza EQ, et al. (2019) relataram alta mortalidade em adultos de $D$. rerio quando expostos à Glib demonstrando que houve diferença de toxicidade entre embriões e larvas quando comparado aos adultos. Markiewicz M, et al. (2017) relataram que a Glib apresentou baixa toxicidade em testes realizados com Daphnia magna, demonstrando como diversos modelos animais são afetados de forma diferente por este antidiabético. A Glib, em comparação aos outros fármacos estudados apresentou efeitos subletais e de teratogenicidade em todas as suas concentrações, sendo considerado o fármaco mais tóxico do nosso estudo. Entretanto apresentou menor efeito sobre a frequência cardíaca. Com isso se observa que Glib provoca mais efeitos morfológicos do que alterações na frequência cardíaca.

Martínez-Viveros EMG, et al. (2018) relataram que Glib provocou efeitos tóxicos em humanos e ratos como dano ao DNA, estresse oxidativo, genotoxicidade e mutagênese; que concentrações na ordem de ng/L induziram oxidação lipídica, proteica e estresse oxidativo no peixe Cyprinus carpio; e que o estresse oxidativo de Glib em peixe pode provocar isquemia cerebral e hipóxia o que poderia justificar os efeitos tóxicos nos animais observados no nosso trabalho. Agora nós observamos diversos efeitos tóxicos a nível subletal e de teratogenicidade em $D$. rerio em concentrações similares a relatada pelo autor e ambientalmente relevantes (TERNES T, et al., 2001).

A baixa taxa de mortalidade relacionada à ausência de batimentos cardíacos também foi observada nos fármacos Glim e Glic. Markiewicz M, et al. (2017) encontrou baixa toxicidade quando expos o microcrustáceo Daphnia magna a Glim e Glic em concentrações ambientais. Mas estudos de ecotoxicidade com Glim e Glic são escassos na literatura (MARKIEWICZ M, et al., 2017).

Simpson SH, et al. (2014) descreveram que os antidiabéticos podem estar relacionados a um aumento no risco de mortalidade por doenças cardiovasculares, sendo a de menor risco a Glic, o antidiabético que apresentou menor toxicidade dentre os estudados neste artigo, mas o único fármaco que apresentou retardo no crescimento dos animais expostos.

Ibrahim IG, et al. (2002) relataram que fetos de ratas diabéticas tratadas com Glic apresentaram malformações como retardo no crescimento. $O$ autor sugere que estes efeitos podem estar relacionados à redução da taxa de absorção de cálcio ou a lesões no fígado. Em relação à exposição química à Glim, os peixes expostos a este fármaco apresentaram efeitos como edemas. Sulfonilureias são conhecidas por causarem efeitos cardiovasculares, o que poderia justificar os nossos resultados (SIMPSON SH, et al., 2014).

\section{CONCLUSÃO}

Os antidiabéticos estudados apresentaram baixa toxicidade em embriões e larvas de $D$. rerio. Esses resultados tentam preencher lacunas de conhecimento a respeito dos efeitos tóxicos dos antidiabéticos sobre o desenvolvimento do zebrafish, uma vez que estudos empregando esses tipos de endpoints ainda são escassos na literatura. Os efeitos observados foram diferentes em relação ao antidiabético e o estágio de desenvolvimento do animal sendo as larvas mais afetadas. Dada a complexidade dos efeitos observados, isto levanta o questionamento de como estes antidiabéticos podem afetar outros grupos animais, uma vez que foram desenvolvidos para apresentar efeitos fisiológicos em mamíferos, e efeitos tóxicos foram encontrados em peixes. Testes crônicos adicionais com o objetivo de avaliar outros parâmetros relevantes como crescimento e comportamento são necessários para compreender outros efeitos tóxicos desses fármacos.

\section{AGRADECIMENTOS E FINANCIAMENTO}

A Universidade Federal Rural de Pernambuco. A CNPq (Conselho Nacional de Desenvolvimento Científico e Tecnológico) (Processo 306947/2020-0) (Processo 423038/2018-4) pelo apoio financeiro. A CAPES (Coordenação de Aperfeiçoamento de Pessoal de Nível Superior) (Processo 88887.485051/2020-00) pelo apoio financeiro. 


\section{REFERÊNCIAS}

1. ALLA LNR, et al. Detection of endocrine disrupting chemicals in Danio rerio and Daphnia pulex: Step-one, behavioral screen. Chemosphere, 2021; 271: 129442.

2. ALAHMAD WR, ALAWI MA. HPLC/ UV/ Fluorescence detection of several pharmaceuticals in sewage treatment plant wastewaters of jordan. Fresenius Environmental Bulletin, 2010; 19(5): 805-810.

3. AL-QAIM F, et al. Detection of Gliclazide in Aqueous Samples using Liquid Chromatography/Time-of-Flight/Mass Spectrometry. Sains Malaysiana, 2016; 45(5): 803-810.

4. BRASIL, Ministério da Ciência, Tecnologia e Inovação. Conselho Nacional de Controle de Experimentação Animal. Resolução Normativa No. 17. Diário Oficial da União, Brasília, 2014; 4: 51-52.

5. BITTENCOURT TQM, et al. Efeitos tóxicos de compostos de vanádio sobre os parâmetros biológicos de embriões e adultos de zebrafish (Danio rerio). Arquivos Brasileiro de Medicina Veterinária e Zootecnia, 2018; 70(6): $1877-1886$.

6. CADENA PG, et al. Protective effects of quercetin, polydatin, and folic acid and their mixtures in a zebrafish (Danio rerio) fetal alcohol spectrum disorder model. Neurotoxicology and Teratology, 2020; 82: e106928.

7. COSTA ECS, et al. Os Efeitos da Metformina sobre a Dispersão do Intervalo QT e QTc de Ratos Diabéticos. Arquivos Brasileiro de Cardiologia, 2008; 90(4): 254-260.

8. FLOREZ JC. The pharmacogenetics of metformin. Diabetologia, 2017; 60(9):1648-1655.

9. FLORY J, LIPSKA K. Metformin in 2019. Clinical Review\& Education, 2019.

10. GODOY AA, et al. Ecotoxicological effects, water quality standards and risk assessment for the anti-diabetic metformin. Environmental Pollution, 2018; 243: 534-542.

11. GHENO EM, et al. Zebrafish in Brazilian Science: Scientific Production, Impact, and Collaboration. Zebrafish, 2016; 13(3): 217-225.

12. HOWE K, et al. The zebrafish reference genome sequence and its relationship to the human genome. Nature, 2013; 496 (7446): 498-503.

13. IBRAHIM IG, et al. Comparative study on the effect of Gliclazide and two Antidiabetic plants used in Folk Medicine on Albino Rat's fetuses. The Egyptian Journal of Hospital Medicine, 2002; 6:80-98.

14. INTERNATIONAL DIABETES FEDERATION. 2019. IDF. Atlas, 9 ed. Disponível em: https://www.diabetesatlas.org/en/resources/. Acessado em: 14 de Junho de 2021.

15. KALRA S, GUPTA Y. Sulfonylureas. Journal of the Pakistan Medical Association, 2015; 65(1):101-4.

16. LAMMER E, et al. Is the fish embryo toxicity test (FET) with the zebrafish (Danio rerio) a potential alternative for the fish acute toxicity test? Comparative Biochemistry and Physiology, 2009; 149: 196-209.

17. MARKIEWICZ M, et al. Ultimate biodegradability and ecotoxicity of orally administered antidiabetic drugs. Journal of Hazardous Materials, 2017; 333: 154-161.

18. MARTÍNEZ-VIVEROS EMG, et al. Environmentally relevant concentrations of glibenclamide induce oxidative stress in common carp (Cyprinus carpio). Chemosphere, 2018; 197: 105-116.

19. NIEMUTH NJ, KLAPPER RD. Low-dose metformin exposure causes changes in expression of endocrine disruptionassociated genes. Aquatic Toxicology, 2018; 195: 33-40.

20. ORGANIZAÇÃO PARA A COOPERAÇÃO E DESENVOLVIMENTO ECONÔMICO (OECD). Test No. 236: Fish Embryo Acute Toxicity (FET) Test, OECD Guidelines for the Testing of Chemicals, Section 2, OECD Publishing, Paris, OCDE, 2013: 1-22.

21. SALES WB, et al. Eficácia da metformina no tratamento do diabetes mellitus gestacional. Revista Brasileira de Pesquisa em Saúde, 2015; 17(3): 133-140.

22. SIMPSON SH, et al. Mortality risk among sulfonylureas: a systematic review and network meta-analysis. The Lancet Diabetes Endocrinol, 2014; 3(1): 43-51.

23. SILVA MCG, et al. The complexation of steroid hormones into cyclodextrin alters the toxic effects on the biological parameters of zebrafish (Danio rerio). Chemosphere, 2019; 214: 330-340.

24. SOUZA EQ, et al. Exposição crônica ao cloridrato de metformina e à glibenclamida causa alterações comportamentais, glicêmicas e de mortalidade em Hemigrammus caudovittatus e Danio rerio. Arquivos Brasileiro de Medicina Veterinária e Zootecnia, 2019; 71(5): 1582-1590.

25. SPIRITA SV, AHILA AAJ. Stages of Embryonic Development of the Zebrafish Danio rerio (Hamilton). European Journal of Biotechnology and Bioscience, 2015;3(6): 6-11.

26. STINCKENS E, et al. An AOP-based alternative testing strategy to predict the impact of thyroid hormone disruption on swim bladder inflation in zebrafish. Aquatic Toxicology, 2018; 200: 1-12.

27. TABASSUM N, et al. Fishing for Nature's Hits: Establishment of the Zebrafish as a Model for Screening Antidiabetic Natural Products. Evidence-Based Complementary and Alternative Medicine, 2015; 2015: 1-16.

28. TERNES T, et al. Determination of neutral pharmaceuticals in wastewater and rivers by liquid chromatographyelectrospray tandem mass spectrometry. Journal of Chromatography A, 2001; 938: 175-185.

29. WEI LV, et al. Mechanisms and Characteristics of Sulfonylureas and Glinides. Current Topics in Medicinal Chemistry, 2020; 20: 37-56.

30. WORLD HEALTH ORGANIZATION. WHO. Classification of diabetes mellitus. WHO Library Cataloguing-in-Publication Data. 2019. Disponível em: https://www.who.int/publications/i/item/classification-of-diabetes-mellitus. Acesso em: 11 de junho de 2021. 